\title{
The Wnt Signalling Pathways: A Short Review and Specific Roles in Bone Biochemistry
}

\author{
Béla Kovács ${ }^{1 *}$, Előd Ernő Nagy², Norbert Nándor Chendrean², Blanka Székely-Szentmiklósi3 ${ }^{3}$ Árpád

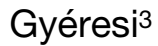

1. University of Medicine and Pharmacy Tîrgu Mureș, Romania

2. Department of Biochemistry and Environmental Chemistry, University of Medicine and Pharmacy Tîrgu Mureș, Romania

3. Department of Pharmaceutical Chemistry, University of Medicine and Pharmacy Tîrgu Mureș, Romania

As musculoskeletal diseases become an emerging healthcare problem worldwide, profound and comprehensive research has been focused on the biochemistry of bone metabolism in the past decades. Wnt signalling, one of the novel described pathways influencing bone metabolism from the early stages of tissue development, has been recently in the centre of attention. Several Wnt ligands are implied in bone forming pathways via canonical ( $\beta$-catenin dependent) and non-canonical ( $\beta$-catenin independent) signalling. Osteoporosis, a catabolic bone disease, has its pathologic background related, inter alia, to alterations in the Wnt signalling, thus key modulators of these pathways became one of the most promising targets in the treatment of osteoporosis. Antibodies inhibiting the activity of endogenous Wnt pathway inhibitors (sclerostin, dickkopf) are recently under clinical trials. The current article offers a brief review of the Wnt signalling pathways, its implication in bone metabolism and fate, and the therapeutic possibilities of osteoporosis through Wnt signalling.

Keywords: Wnt signalling, bone metabolism, osteoporosis, autophagy, antibodies

Received 14 June 2017 / Accepted 10 July 2017

\section{Introduction}

Osteoporosis is characterised by a loss in both bone mass and resistance, as a result of a disruption between bone mineral mass and perturbation of its microarchitecture, emerging in pronounced bone fragility. The pathophysiology of osteoporosis has a complex and multifactorial aetiological background, skeletal fragility having its origin from an inappropriate skeletal development or pronounced bone resorption as a result of microarchitecture deterioration, or inadequate remodelling after resorption $[1,2]$. Musculoskeletal diseases represent one of the leading global economic burdens, affecting more than five million people in Europe and hundreds of millions worldwide, mainly postmenopausal women [3].

Although Wnt signalling is a cellular mechanism known only since a few decades, yet, the Wnt related processes are highly conserved universal pathways, dating from the appearance of the first metazoan species, around 650 million years ago, in the Precambrian Era [4]. Structurally, the Wnt ligands are hydrophobic glycoproteins, comprising of approximately 350 amino acids, with 23-25 cysteine residues, providing the possibility to form intra-, or inter-molecular disulphide bonds [5,6]. Currently, three important Wnt signalling pathways are described, in the mechanisms of which several Wnt ligands, receptors, modulators and effectors are implied.

Hereby we present a short overview of the Wnt pathways, regulations, antagonists and therapeutic possibilities for osteoporosis targeting Wnt signalling-related molecules.

\section{The Wnt/ $\beta$-catenin pathway}

$\beta$-catenin (cadherin-associated protein, beta) plays a cardinal role in the Wnt signalling pathway and cellular mechanisms. In the "OFF" state of the canonical (Wnt/ $\beta$ catenin) signalling the effector molecule is phosphorylated by a hetero-tetramer destruction complex, under the action of adenomatous polyposis coli (APC), axin, glycogen synthase kinase 3-beta (GSK-3 $\beta$ ) and casein-kinase I-alpha $(\mathrm{CK}-\mathrm{I} \alpha)$. The phosphorylation of $\beta$-catenin is realized on four different hydroxyl amino acid sites: at $\operatorname{Ser}^{33}, \operatorname{Ser}^{37}$, Thr $r^{41}$ by GSK-3 $\beta$ and in position Ser ${ }^{45}$ by CK-I $\alpha$. This way, the protein becomes a target for ubiquitination and a subsequent degradation inside a proteasome [7-9].

In the presence of Wnt ligands, Frizzled (Fz) and Lipoprotein receptor related protein (LRP) 5 or 6 receptors are stimulated and undergo hetero-dimerization. Fz are large receptors, comprised of seven transmembrane sites and a cysteine rich domain (CRD), which provides a docking site for the Wnt ligands. This interaction results in a subsequent phosphorylation of the LRP receptors, which in turn represents an adherence site for axin via dishevelled protein (Dsv). The phosphorylation process takes place simultaneously for both LRPs (by CK-I $\gamma$ and GSK-3 $\beta$ ), and Dsv (by CK-IE, CK-II). These conformational transformations also lead to an over-expressed activity of Dsv on the GBP/ FRAT complex (GSK-3 $\beta$ binding protein) and antagonize the effect of the enzyme. Thus, the destruction complex undergoes modifications which lead to its disintegration. As a result, the intra-cytoplasmic $\beta$-catenin levels rise and with its transposition into the nucleus, the transcription of several genes occur [7-8, 10-11]. 
Table I. Components of the Wnt signalling pathways [33]

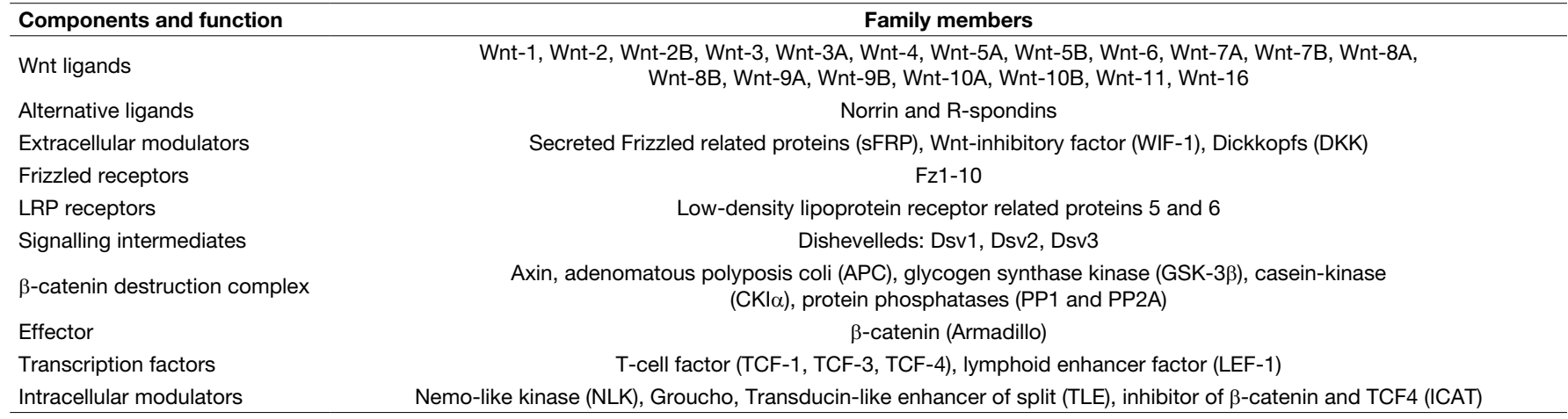

\section{The Wnt/Ca ${ }^{2+}$ signalling pathway}

Although $\beta$-catenin plays a primordial role in the Wnt signalling pathways, several alternative routes have been described. Considering that $\mathrm{Fz}$ receptors are G-protein coupled, this could lead to an interaction with second messenger molecules. The activation of $\mathrm{Fz}$ receptors by "non-canonical" Wnts enrolls enhanced activity of inositol-4,5-triphosphate $\left(\mathrm{IP}_{3}\right)$ and diacyl-glycerol (DAG). Elevated levels of these second messenger molecules result in higher intra-cytoplasmic calcium concentrations. Calcium in relation with calmodulin $(\mathrm{CaM})$ activates the calcium-calmodulin-kinase II (CaMK-II). Calcium could also interact with DAG and $\mathrm{IP}_{3}$. On one hand the calciumDAG complex results in protein-kinase $\mathrm{C}$ activation, on the other hand, calcium- $\mathrm{IP}_{3}$ complex interacts with calcineurin (Can). Subsequently, Can activates nuclear factor associated with T-cells (NFAT), resulting in gene expression in several tissues [12]. Recent findings describe that, despite the current standpoints - that the canonical and non-canonical pathways do not interact and have antagonistic effects - there is a relation between these signalling routes, acting in an interdependent way. Considering that $\beta$-catenin, a $92 \mathrm{kDa}$ protein, has limited or no pass through the nuclear envelope due to its size, this limitation could be

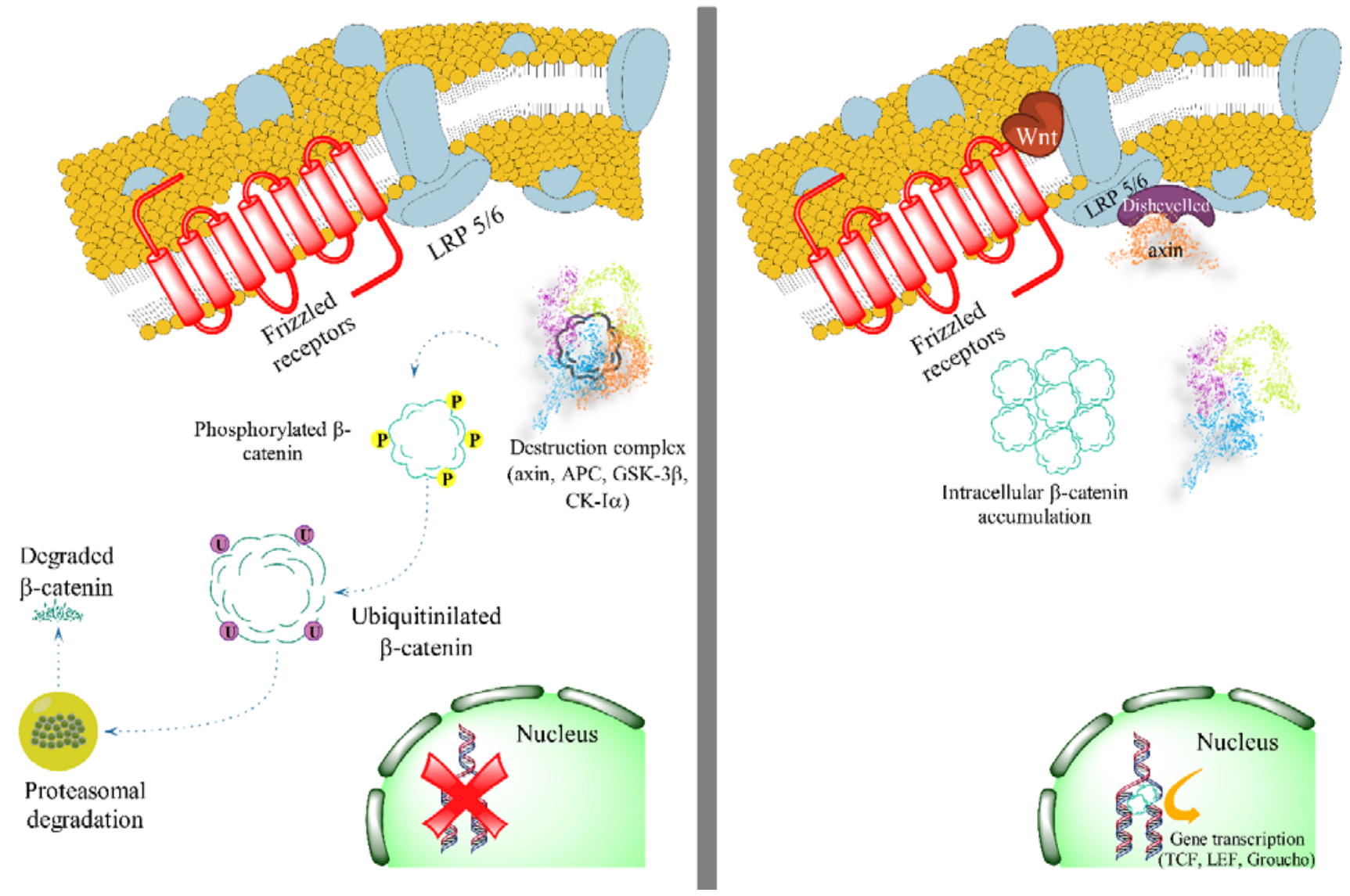

Fig. 1. $\beta$-catenin dependent Wnt signalling - left: in the absence of Wnt ligands the degradation of $\beta$-cathenin occurs inside a destruction complex; right: contrarily, in the presence of Wnt ligands, the intra-cytoplasmic accumulation of $\beta$-cathenin results in gene transcription. Abbreviations: LRP - low-density lipoprotein receptor related proteins, APC - adenomatous polyposis coli, CKl $\alpha$ - casein kinase l $\alpha$, GSK$3 \beta$ - glycogen synthase kinase $3 \beta$, Dsv: dishevelled, TCF - T-cell factor, LEF - lymphoid enhancer binding factor 
overpassed by the interrelation of Wnt/ $\beta$-catenin - Wnt/ $\mathrm{Ca}^{2+}$ pathways. The increase of intra-cytosolic $\mathrm{Ca}^{2+}$ could possibly lead to nuclear barrier depolarization and subsequent $\beta$-catenin translocation into the nucleus [13]. The relation between these is also emphasized by the activation of Nemo-like kinase (NLK) by $\mathrm{Ca}^{2+}$ signalling, which hereinafter phosphorylates T-cell factor (TCF), thus acting like an inhibitor for the $\beta$-catenin activity [7].

\section{Wnt/JNK pathway}

The Wnt/JNK (Jun-N-terminal kinase) or PCP (planar cell polarity) pathway involves also the action of Dsv proteins, mainly its PDZ, DEP domains, which is capable to activate on a downstream route rhoA or cdc42 (cell division cycle protein). Wnt activation of Fz receptors results in a complex formation between Dsv and Daam-1 (Dishevelled associated activator of morphogenesis 1). This complex subsequently activates Rho-GTPases via WGEF (Rho-guanine exchange factor). This leads to the activation of ROCK (rho-associated kinase), resulting in phosphorylation of the myosin light chain, thus creating the interaction between actin and myosin, leading to the disruption of the cytoskeleton's integrity. Alternatively, the activation of Dsv DEP domain results in Rac-GTPase activation. These GTPase type proteins subsequently activate JNKs $[7,14]$. Among these JNK1 and JNK2 are ubiquitously present, whereas JNK3 is expressed mainly in the brain. Various types of proteins are targets for the JNKs, mainly transcription factors, which are responsible for cell proliferation or cell apoptosis (ATF2 - activating transcription factor 2; Bcl-2 protein - B-cell lypohoma-2; BAD - Bcl2 associated death promoter) [15]. Interactions between the JNK and $\beta$-catenin pathways have also been described. Activation of Rac1-GTPase and JNK2 could result in the phosphorylation of the free intra-cytoplasmic $\beta$-catenin, thus intensifying its translocation in the nucleus [9].

\section{Regulation of the Wnt signalling pathways}

Although $\beta$-catenin plays a primordial role in Wnt signalling, several ligands with regulatory activity are known to influence the previously described pathways. Norrins, are proteins with a cysteine-knot motif, with special affinity to Fz4 receptors and LRP5 as co-receptors, and activate the canonical Wnt signalling pathways. The activity of Norrins was described in the vascular development in the eye, ear, brain and female reproductive organs [16].

The proteins of the R-spondins (RSPO) family have 234 to 272 amino acids and present the following domains in their structure: an $\mathrm{N}$-terminal putative signal sequence for secretion, a thrombospondin type I repeat (TSR) domain, a variable length domain, rich in basic amino acids and a furine-like CRD. RSPOs are also considered as Wnt agonists, as they activate the $\beta$-catenin signalling pathway, however RSPOs may have different receptors as Wnts [17]. Recently, the presence of leucine-rich repeat containing Gprotein-coupled receptors (LGR) has been described. The activation of these receptors could lead to an enhanced and more potent activity, showing synergy with the $\beta$-catenin and PCP pathways [18]. RSPOs are potential ligands for the Kremen (Krm) receptors, acting like competitive an-

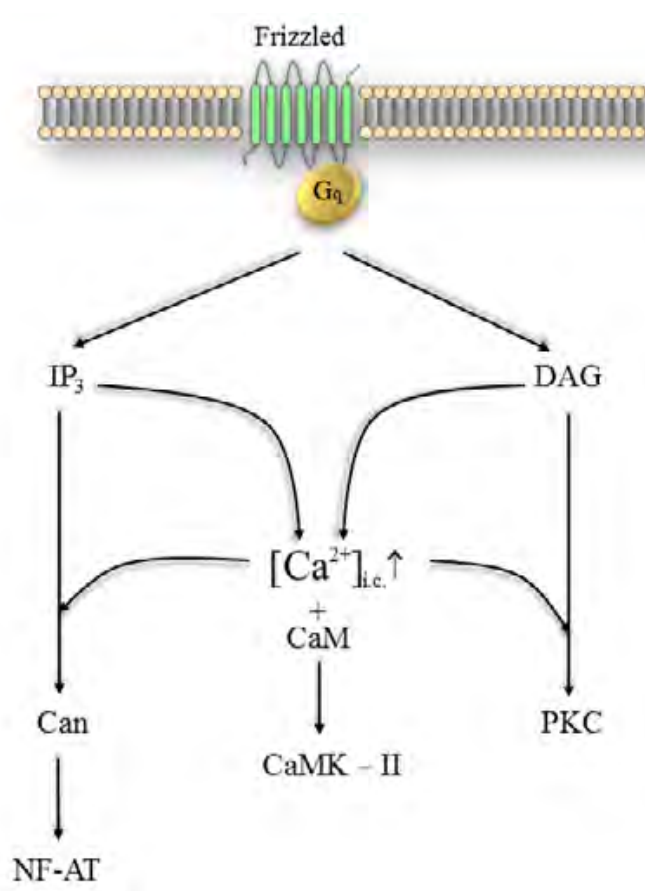

Wnt/Ca ${ }^{2+}$ signalling pathway

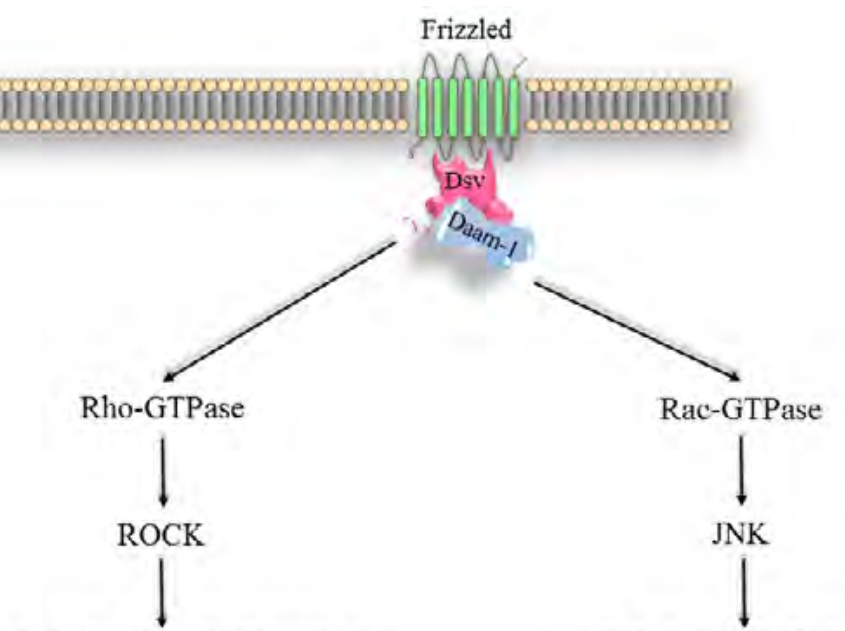

Cytoskeleton disintegration

Wnt/JNK signalling pathway

Fig. 2. Brief overview of the "non-canonical" Wnt signalling pathways. Abbreviations used: IP3 - inositol triphosphate, DAG - diacylglycerol, Can - calcineurin, PKC - protein kinase C, CaM - calmodulin, CaMK-II - calcium calmodulin kinase II, NF-AT - nuclear factor associated with T-cells, Dsv - dishevelled, Daam-1 - Dishevelled associated activator of morphogenesis 1, ROCK - rho associated kinase, JNK - Jun-N terminal kinase, ATF-2 - activating transcription factor 2, Bcl-2 - B cell lymphoma, Bad - Bcl-2 associated death promoter 
tagonist for Dickkopfs (DKK), therefore inhibiting the formation of DKK/Krm complex [9].

Soluble Frizzled-related proteins (sFRP) are antagonists of the Wnt signalling pathways. They form complexes not only with Wnts, but also form non-functional complexes with $\mathrm{Fz}$ receptors. This results in an inhibitory mechanism for the $\beta$-catenin and/or PCP pathways. It has also been described that sFRPs do not always act like antagonists, as in some cases activation of Wnt signalling could be promoted. This could be explained in such a way that sFRPs have low- and high-affinity binding sites for the Wnt proteins. When binding to the low-affinity site, sFRPs behave as inhibitors, contrarily, binding to the high-affinity site they would behave as activators [19].

WIF (Wnt inhibiting factor), unlike sFRPs binds only to the Wnt proteins, exerting an inhibitory effect on the canonical, as well as on the non-canonical pathway. Beside this, WIF has the potential to modulate and regulate extracellular proteins which are implicated in Wnt signalling.

Members of the Dickkopfs (DKK) family are considered as specific Wnt pathway inhibitors. DKKs are competitive antagonists of the LRP receptors and in this way inhibit the formation of the Fz-LRP complex. It is also believed that DKKs could disrupt the Wnt-Fz complex. Besides, DKKs have the potential to bind to Krm receptors, behaving as agonists. Krm receptors provide the internalization of the LRP receptors and prohibit the possibility of FzLRP receptor formation [9].

Other inhibitory mechanisms in the Wnt signalling are represented by Shisa proteins, which suppress Fz receptor docking to the cell membrane, by trapping the receptors inside the endoplasmic reticulum. Cerberus, Nodal, bone morphogenetic protein (BMP), insulin-like growth factor binding protein (IGFBP-4) inhibit Wnt and both Fz and LRP receptors [9].

\section{Wnt ligands in bone metabolism and fate}

Several Wnt ligands are implied in osteoblastogenesis. Wnt1 was shown to enhance $\beta$-catenin mediated osteoblast formation, by an endogenous phosphorylation of GSK-3 $\beta$ via G-protein coupled Fz receptors and LRP 5 or LRP 6 co-receptors, which leads to an increased $\beta$-catenin activity. Furthermore, it was elucidated that the enhanced activity of the Wnt canonical pathway results in an expressed Runx2 downstream regulation [20]. The downstream osteoblastogenetic and mineralization enhancing properties of Wnt1 were also described in Wisp1 $1^{-/}$mice. Wisp1/CCN4 (Wnt1-inducible signalling pathway protein $1 /$ connective tissue growth factor) induces mesenchymal stem cell differentiation and osteoblast proliferation and maturation in the detriment of chondrocyte differentiation. It was also described that Wisp1 interacts with BMP-2 having a positive effect on osteogenesis. Wisp1 proved to be a key regulator of bone turnover and Wnt signalling, resulting a significantly lower bone mass, with diminished biomechanical strength in Wisp-1 knockout mice [21-23]. Wollnik et al. recently reported that different mutations, hypofunctional alleles of Wnt1 may lead to osteogenesis imperfecta, or low-bone-mass phenotype which predisposes to an early-onset of osteoporosis. These altered molecular changes in Wnt1 protein result in a loss of activity over LRP5-receptor mediated $\beta$-catenin signalling [24]. Similar mutations were identified by other research groups in families with hereditary early-onset osteoporosis and osteogenesis imperfecta [25]. Okamoto et al. demonstrated the importance of non-canonical Wnts during osteoblastogenesis. In calvarial cell cultures increased levels of mRNA expression of Wnt5a and Wnt10b were observed, but not Wnt7b. Furthermore, it was described that Wnt5a, as a non-canonical Wnt ligand, induces the expression of LRP5 and LRP6 receptors during osteoblast differentiation, thus being implied in Wnt/ $\beta$-catenin dependent, canonical stimuli of osteoblasts [26]. Contrarily, Chen et al. described that Wnt7b is involved in bone formation via mTORC1 (mammalian target of rapamycin complex 1) activation. The bone formation activity of Wnt7b was assessed by the measurement of serum osteocalcin levels, which appeared to be significantly higher in comparison to the control group. Also, CTX-I (C-terminal telopeptide) levels showed a similarity with the control group in two months old mice [27]. Wnt11 and Wnt16 are also key regulators of bone homeostasis. Wnt11 acts through the activation of R-spondin2, a known activator of the Wnt signalling. Moreover, Friedman et al. demonstrated enhanced alkaline phosphatase activity in murine pre-osteoblasts under Wnt $11^{+}$over-expression. Wnt11 also proved to be a potent enhancer of BMP2-induced osteoblast maturation and mineralization [28]. Wnt16, known as an activator for both canonical and non-canonical Wnt signalling, has a great role in bone thickness and porosity modulation, interestingly not affecting in a significant manner osteoblast differentiation, proliferation and maturation, contributing modestly to bone homeostasis. Altering the RANKL/RANK/OPG triad, Wnt16 contributes to an OPG over-expression, potentiating the inhibition of RANKL-induced osteoclastogenesis, interfering with osteoblast-to-osteoclast cross talking [29].

\section{Wnt signalling and autophagy in bone}

Autophagy is one of the cell's novel described fate mechanisms, involved in tissue regeneration and recycling of molecules of key importance in cell metabolism (macromolecules and eventually organelles). Onal et al. reported that autophagy suppression in osteocytes results in decreased bone mass and bone turnover in mice and proves to contribute to the state of oxidative stress, thus resulting in a process similar to ageing in bone tissue and skeleton. Sustaining evidence was published by Nollet et al., demonstrating that impaired autophagy in osteoblasts and the environmental oxidative stress may lead to an enhanced osteoclastogenesis via TNFSF-11 activation (RANKL) 
and leading to osteoporotic-like phenotype [30,31]. Autophagy negatively alters the $\mathrm{Wnt} / \beta$-catenin cascade by promoting the degradation of all three isoforms (Dsv1, Dsv2 and Dsv3) of docking protein, Dishevelled. This attenuation in the Wnt signalling was reported by Gao et al. in autophagy-deficient cells (Atg $5^{-/-}$and $A \operatorname{tg} 7^{-/}$). It was also shown that inappropriate autophagy mechanisms lead to a slower turnover rate of all Dsv members [32].

\section{Molecular targets in Wnt signalling}

Sclerostin (Wise/SOST) is an osteocyte-expressed glycoprotein, inhibiting the bone forming activity of osteoblasts, by interrupting the Wnt signalling. SOST selectively inhibits the interaction between the Fz and LRP5 or LRP6 receptors by binding through a PNAIG motif to the receptor complex and disrupts its functionality. From the beginning of the millennium SOST has been one of the promising targets for the treatment of osteoporosis. As SOST is not ubiquitously expressed, its effects are only limited to bone metabolism; thus its inhibition using targeted monoclonal antibody therapy, will not result in systemic effects as in the case of other antagonists of different Wnt inhibitors [34,35]. Sclerostin being a physiological antagonist of osteoblast operated bone matrix formation, osteoporosis can be treated by its neutralization using monoclonal antibodies. Several antibodies were already developed and are currently in clinical studies, such as blosozumab, which is described as an immunoglobulin G4 (IgG4) protein, or romosozumab, formally known as AMG785 [36]. Blosozumab is a humanized monoclonal antibody targeted against sclerostin, which in phase II clinical trials produced significant changes in postmenopausal women, mostly in lumbar spinal and hip bone mass density (BMD). Romosozumab, similarly to blosozumab is a monoclonal antibody that inhibits the effects of the sclerostin, increases osteoblast mediated bone formation and decreases the intensity of bone resorption. In a study made by Cosman et al., after 12 weeks of treatment with romosozumab followed by another 12 months with denosumab (monoclonal antibody targeted against RANKL), postmenopausal women with osteoporosis had a $73 \%$ lower risk of vertebral fracture in comparison to the control group. Romosozumab increased BMD T score of the lumbar spine and the hip bone after 6 months of treatment, while after 12 months the increase was even more significant [37]. In another study made by Sugiyama et al. made upon romosozumab and blosozumab, there were no significant changes in the areal BMD of the radius bone which reflects that the forearm is not exposed to high levels of mechanical strain under normal physical activity and therefore the anti-sclerostin antibodies could not increase the strength and density of the bones which are not exposed to stress and strains within the skeleton. The treatment with anti-sclerostin antibodies also has its limitations, in both cases postmenopausal women's increased areal $\mathrm{BMD}$ returned to the pre-treatment levels within a year despite the continued therapy with romosozumab or blosozumab [38].

DKK-1 is the most studied protein of the four members of the DKK family (DKK-1 to DKK-4). DKK-1 is a secreted antagonist of the $\mathrm{Wnt} / \beta$-catenin signalling, which inhibits the Wnt-induced stabilization of $\beta$-catenin and $\beta$-catenin/Tcf-dependent transcription by binding to LRP 5 or 6 . Besides this blocking mechanism, DKK-1 also has effects on the Wnt/LRP6 signalling by disrupting it and promoting endocytosis via Krm-1 and $\mathrm{Krm}-2$. BHQ880 is an anti-DKK-1 monoclonal IgG1 isotype antibody developed for the potential treatment of multiple myeloma (MM) produced osteolytic bone fractures, where the neutralizing effect of the antibodies could readjust the disrupted balance between bone formation and bone resorption. The drug encoded BHQ880, in vitro increased the osteoblast differentiation and serum human osteocalcin levels, neutralized the negative effects of MM cells on osteoblastogenesis, reduced interleukin-6 (IL-6) secretion, and in addition up-regulated $\beta$-catenin levels and down-

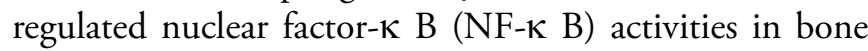
marrow stromal cells $[39,40]$. In a preclinical model study published by Goldstein et al, BHQ880 reduced DKK-1 serum levels in treated mice with orthotopically implanted patient-derived osteosarcoma tumours, slowed the growth of the tumours and inhibited metastasis by neutralizing the DKK-1 protein, which is overexpressed by these cells. The positive effects over the tumorous cells were correlated with increased $\beta$-catenin levels and increased expression of the bone differentiation marker osteopontin, which altogether suggests that Wnt signalling has antitumorigenic effects [40].

PF-04840082 is a humanized prototype anti-DKK-1 IgG2 isotype antibody for the potential treatment of osteoporosis that binds the physiological antagonist of the Wnt/LRP5 signal pathway, therefore increasing bone mass and BMD by activating osteoblasts. In 2010 Betts et al. published a study about the pharmacokinetic/pharmacodynamic modelling of the antibody in concordance to dose and effect that shows significant decreasing in free DKK-1 concentrations in rats and cynomolgus monkeys, with a proper safety and efficiency interval that can be transposed to humans, making it a potentially effective biological drug after furthermore preclinical and clinical studies [41].

Many antibodies that bind and neutralize either sclerostin or DKK-1 are in preclinical or clinical trials making them a potential treatment for postmenopausal osteoporosis and MM induced disease in the future. Anti-sclerostin and anti-DKK-1 antibodies have the potential to improve BMD T score, fracture healing via increased bone formation. However anti-sclerostin treatment alone is currently not enough, patients presented a relapse in BMD levels after a year despite the still undergoing therapy with the antibodies [38,42]. Therefore Florio et al. hypothesized that blocking both proteins at once with one antibody, this could further increase Wnt signalling, resulting in a 
more robust effect on bone formation and repair. Their results show that the simultaneous inhibition of sclerostin and DKK-1 by the newly engineered bi-specific heterodimer IgG antibody promotes synergistic bone formation in mice. The inhibition alone of sclerostin leads to increased DKK-1 levels, which act as a negative regulator upon the bone formation, reducing the administered anti-sclerostin antibody's effects. The inhibition of either one of them may stimulate compensatory mechanisms that would rise the other antagonist levels in order to return the Wnt signalling in a steady state, making the dual inhibition a more proper treatment of osteoporosis in the future [42].

\section{Conflict of interest}

None to declare.

\section{References}

1. Raisz L - Pathogenesis of osteoporosis: concepts, conflicts and prospects, J Clin Invest, 2005; 115:3318-3325

2. Rizzoli R - Atlas of Postmenopausal Osteoporosis, Current Medicine Group, London, United Kingdom, 2010

3. Svedbom A, Hernlund $E$, Ivergård $M$ et al. - Osteoporosis in the European Union: a compendium of country-specific reports, Archives of Osteoporosis, 2013, 8:137

4. Gordon MD, Nusse R - Wht Signalling: Multiple Pathways, Multiple Receptors, and Multiple Transcription Factors, J BiolChem, 2006; 281:22429-22433

5. Mikels AJ, Nusse R - Wnts as ligands: processing, secretion and reception, Oncogene, 2006; 25:7461-7468

6. Coudreuse D, Korswagen $\mathrm{HC}$ - The making of Wnt: new insights into Wnt maturation, sorting and secretion, Development, 2007; 134:3-12

7. Kestler HA, Kühl M - From individual Wnt pathways towards a Wnt signalling network, Philos Trans R Soc Lond B Biol Sci, 2008; 363:13331347

8. Rao TP, Kühl M - An Updated Overview on Wnt Signaling Pathways A prelude for more, Cric Res, 2010; 106:1798-1806

9. MacDonald BT, Tamai $\mathrm{K}, \mathrm{He} X-W n t / \beta$-catenin signaling: components, mechanisms, and diseases, Developmental Cell, 2009; 17:9-26

10. Clevers $\mathrm{H}-$ Wnt/ $\beta$-Catenin Signalling in Development and Disease, Cell, 2006; 127:469-480

11. Logan CY, Nusse R - The Wnt Signalling Pathway in Development and Disease, Ann Rev Cell Dev Biol, 2004; 20:781-810

12. De A - Wnt/Ca2+ signalling pathway: a brief overview, Acta Biochimica et Biophysica Sinica, 2011; 43:745-756

13. Thrasivoulou C, Millar M, Ahmed A - Activation of intracellular calcium by multiple Wnt ligands and translocation of $\beta$-catenin into the nucleus: a convergent model of $\mathrm{Wnt} / \mathrm{Ca} 2+$ and $\mathrm{Wnt} / \beta$-catenin pathways, J Biol Chem, 2013; 288:35651-35659

14. Komiya $Y$, Habas R - Wnt signal transduction pathways, Organogenesis, 2008, 4:68-75

15. Saadeddin A, Babaei-Jadidi R, Spencer-Dene B et al. - The Links between Transcription, $\beta$-catenin/JNK Signaling and Carcinogenesis, Molecular Cancer Research, 2009; 7:1189-1196

16. Kikuchi A, Yamamoto $H$, Kishida S - Multiplicity of the interactions of Wnt proteins and their receptors, Cell Signal, 2007; 19:659-671

17. Jin YR, Yoon JK - The R-spondin family of proteins: emerging regulators of Wnt signalling, Int J Biochem Cell Biol, 2012; 44:1055-1061

18. Glinka A, Dolde C, Kirsch N et al. - LGR4 and LGR5 are R-spondin receptors mediating $\mathrm{Wnt} / \beta$-catenin and $\mathrm{Wnt} / \mathrm{PCP}$ signalling, EMBO reports, 2011; 12:1055-1061

19. Kawano Y, Kypta R - Secreted antagonists of the Wnt signalling pathway, J Cell Sci, 2003; 166:2627-2634
20. Sung-Ho K, Jung SH, Jeong-Chae L - Crucial roles of canonical Runx2dependent pathway on Wnt1-induced osteoblastic differentiation of human periodontal ligament fibroblasts, Mol Cell Biochem, 2015; 402:213-223

21. Maeda A, Ono M, Holmbeck K et al. - WNT1-induced Secreted Protein-1 (WISP1), a Novel Regulator of Bone Turnover and Wnt signalling, J Biol Chem, 2015; 290:14004-14018

22. French DM, Kaul RJ, D'Souza AL - WISP-1 Is an Osteoblastic Regulator Expressed During Skeletal Development and Fracture Repair, Am J Pathol, 2004; 165:855-867

23. Mitsuaki O, Colette Al, Tina MK et al. - WISP-1/CCN4 Regulates Osteogenesis by Enhancing BMP-2 Activity, J Bone Miner Res, 2011; 26:193-208

24. Wollik B et al. - Mutations in WNT1 Cause Different Forms of Bone Fragility, Am J Hum Genet, 2013; 92:565-574

25. Laine CM, Joeng KS, Campeau P et al. - WNT1 Mutations in Early-Onset Osteoporosis and Osteogenesis Imperfecta, N Engl J Med, 2013; 368, 1809-1816

26. Okamoto $M$, Udagawa $N$, Uehara $S$ et al. - Noncanonical Wnt5a enhances Wnt/ $\beta$-catenin signalling during osteoblastogenesis, Nature Scientific Reports; 2014, 4:4493

27. Chen J, Tu X, Esen E et al. - Wnt7B Promotes Bone Formation in part through mTORC1, PLOS Genetics, 2014; 10:1-13

28. Friedman MS, Oyserman SM, Hankenson KD - Wnt11 Promotes Osteoblast Maturation and Mineralization through R-spondin 2, J Biol Chem, 2009; 284:14117-14125

29. Gori F, Lerner U, Ohlsson C et al. - A new Wnt on the bone: Wnt16, cortical bone thickness, porosity and fractures, Nature BoneKEy Reports; 2015; 4:1-6

30. Onal M, Piemontese $\mathrm{M}$, Xiong $\mathrm{J}$ et al. - Suppression of Autophagy in Osteocytes Mimics Skeletal Aging, J Biol Chem, 2013; 288:1743217440

31. Nollet M, Santucci-Darmanin S, Breuil V et al. - Autophagy in osteoblasts is involved in mineralization and bone homeostasis, Autophagy, 2014; 10:11:1965-1977

32. Gao C, Cao W, Bao L et al. - Autophagy negatively regulates Wnt signalling by promoting Dishevelled degradation, Nature Cell Biology, 2010; 12:781-790

33. Baarsma HA, Königshoff M, Gosens R - The Wnt signalling pathway from ligand secretion to gene transcription: Molecular mechanisms and pharmacological targets, Pharmacol Ther, 2013; 138:66-83

34. Holdsworth G, Slocombe P, Doyle C et al. - Characterization of the Interaction of Sclerostin with the Low Density Lipoprotein Receptorrelated Protein (LRP) Family of Wnt Co-receptors, J Biol Chem, 2012; 287:26464-26477

35. Minisola G, Iuliano A, Prevete I - Emerging therapies for osteoporosis, Reumatismo, 2014; 66:112-124

36. MacNabb C, Patton D, Hayes JS - Sclerostin Antibody Therapy for the Treatment of Osteoporosis: Clinical Prospects and Challenges, Journal of Osteoporosis, 2016; 1-22

37. Cosman F, Crittenden DB, Adachi JD et al. - Romosozumab Treatment in Postmenopausal Women with Osteoporosis, N Eng J Med, 2016; 375:1532-1543

38. Sugiyama T, Torio T, Miyajima T et al. - Romosozumab and blosozumab: alternative drugs of mechanical strain-related stimulus toward a cure for osteoporosis, Frontiers in Endocrinology, 2015; 6:1-4

39. Fulciniti M, Tassone P, Hideshima T et al. - Anti-DKK1 mAb (BHQ880) as a potential therapeutic agent for multiple myeloma, Blood Journal, 2009; 114:371-379

40. Goldstein SD, Trucco M, Guzman WB et al. - A monoclonal antibody against the Wnt signaling inhibitordickkopf-1 inhibits osteosarcoma metastasis in a preclinical model, 2016; Oncotarget, 7:21114-21123

41. Betts AM, Clark TH, Yang J et al. - The application of target information and preclinical pharmacokinetic/pharmacodynamic modelling in predicting clinical doses of a Dickkopf-1 antibody for osteoporosis, 2010, J Pharmacol Exp Ther, 333:2-13

42. Florio M, Gunasekaran K, Stolina M - A bispecific antibody targeting sclerostin and DKK-1 promotes bone mass accrual and fracture repair, Nature Communications, 201; 7:1-14 